\title{
Lively data: discover, browse and access ocean altimetry data on internet
}

\author{
V. Rosmorduc ${ }^{1}$, T. Jolibois ${ }^{2}$, and O. Lauret ${ }^{3}$ \\ ${ }^{1}$ Collecte Localisation Satellites (CLS), 8-10 rue Hermes, 31520 Ramonville, StAgne, France \\ ${ }^{2}$ Coframi, Toulouse, France \\ ${ }^{3}$ Silogic, Toulouse, France
}

Received: 15 September 2005 - Revised: 10 February 2006 - Accepted: 5 March 2006 - Published: 6 June 2006

\begin{abstract}
The Products and Services (P\&S) department in the Space Oceanography Division at CLS (Collecte, Localisation, Satellites) is in charge of distributing and promoting altimetry and operational oceanography data. The department is thus involved in the Aviso satellite altimetry project (the French service which distributes altimetry products since 1992), in the Mercator ocean operational forecasting system, and in the European Godae/Mersea ocean portal. Aiming to a standardisation and a common vision and management of all these ocean data, all these projects, led to the implementation of several Opendap/LAS Internet servers (Baudel et al., 2004). Some of the possibilities of the tools, as well as how-to information will be highlighted, as they are in the "Lively data" section of Aviso website (see http://www.aviso.oceanobs.com/html/donnees/las/). Moreover, with a two-year experience we now have some feedback and analysis of how people - users, would-be users and students alike - are using this tool, some ideas for possible enhancements, etc.
\end{abstract}

\section{Altimetry \& Aviso}

Space altimetry is a technique that measure sea surface height by satellite. Level 2 data, Geophysical Data Record, are along-track products with the complete set of measurements, auxiliary data and corrections, and thus quite hard to use. Higher-level data were therefore designed to help users handle altimetry data. However, even with gridded data some new users have problems - mostly programming difficulties. Moreover, even expert users often want a "quick-look" view on a dataset. Such easiness to create plots and visualise data is also a major asset for education (to help student in getting familiar with satellite data) and for outreach, too (to show in-

Correspondence to: V. Rosmorduc

(vinca.rosmorduc@cls.fr) terested people - including would-be users - what the data can "look" like). Thus interactive visualization is clearly a need.

Aviso stands for Archiving, Validation and Interpretation of Satellite Oceanographic data. It was set up in 1992 to process, archive and distribute data from the NASA (National Aeronautics and Space Administration)/CNES (Centre National d'Études Spatiales) ocean radar altimetry satellite Topex/Poseidon (T/P). Now, thirteen years later, Aviso user service's activities encompass (Rosmorduc, 2005):

- Operational distribution of Topex/Poseidon, Jason-1 and Envisat Geophysical Data Records (GDRs);

- Distribution of high-level altimetry products, including operational distribution of Ssalto/Duacs near-real time products;

- A catalogue of altimetry, orbit determination and precise location products;

- Outreach of ocean altimetry, orbit determination and precise location activities.

All information, whether on data, the missions or outreach are updated on the Aviso website (http://www.aviso. oceanobs.com), in both French and English (a Spanish section is also available, for basic information, but not updated).

During those years, many changes occurred. The major change in data distribution has come from Internet. Online distribution became possible around 1995, and feasible (at least for developed countries) about 1999-2000 for important amounts of data with high bandwidth Internet. In the same time, data format standardisation was progressing. Moreover, interactive tools have now reached a maturity that enable a centre like Aviso to use them for its data. Thus interactive access and visualization of altimetry data is possible.

Published by Copernicus GmbH on behalf of the European Geosciences Union. 


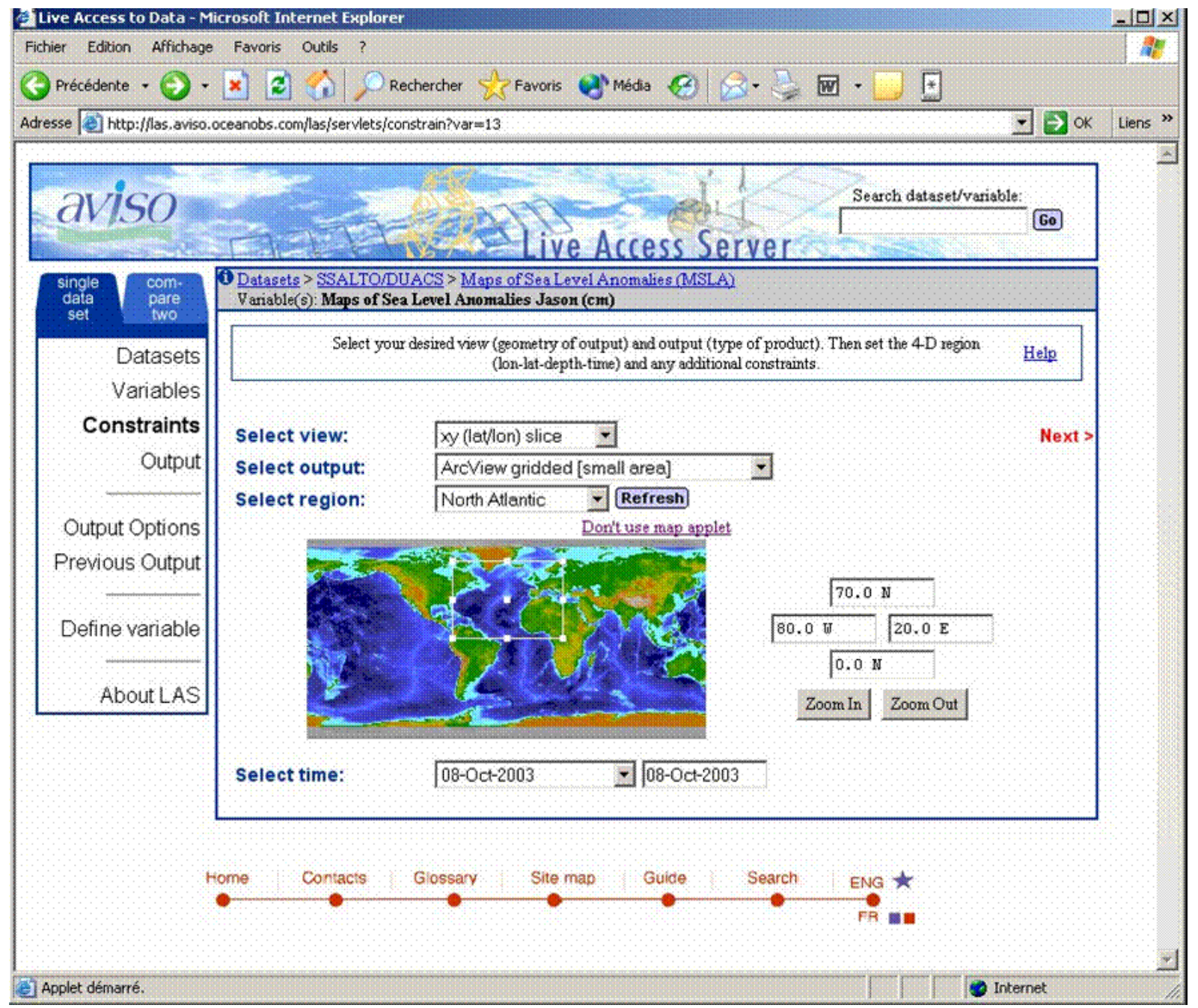

Fig. 1. Aviso Live Access Server.

\section{Opendap \& Live Access Server}

Aviso opened an Opendap and a Live Access Server in 2003 (http://opendap.aviso.oceanobs.com; http://las.aviso. oceanobs.com). The Opendap enables access to remote data sets through familiar data analysis and visualization packages, whereas the Live Access Server is an interactive online visualization tool for plotting gridded data. This choice was made in the Godae and Mersea international operational oceanography project frame.

\subsection{What are Opendap and LAS?}

Opendap (Open source Project for a Network Data Access Protocol, a.k.a. DODS, see http://opendap.org/) handles transport, translation and extracting subsets of data. It allows you to access remote data over the internet:

- using familiar data analysis and visualization packages like Matlab, Ferret, IDL,... as if the data were on your computer (see http://opendap.org/developers/ third_party_software.html)

- without worrying about data storage formats, translating them on-the-fly (including in ascii), if necessary

The Live Access Server (or LAS) is an online graphical interface designed to provide flexible access to geo-referenced scientific data. It can present distributed data sets as a unified virtual data base through the use of Opendap networking (http://ferret.pmel.noaa.gov/Ferret/LAS). Versatile and interactive, it can be used for plotting and retrieving gridded data. The use is rather intuitive for non-expert public, and does not need data-plotting dedicated software to be installed on the user's computer. LAS enables the Web user to:

- visualize data with on-the-fly graphics (plotting maps, but also longitude-time or along-time evolution at one point...), 


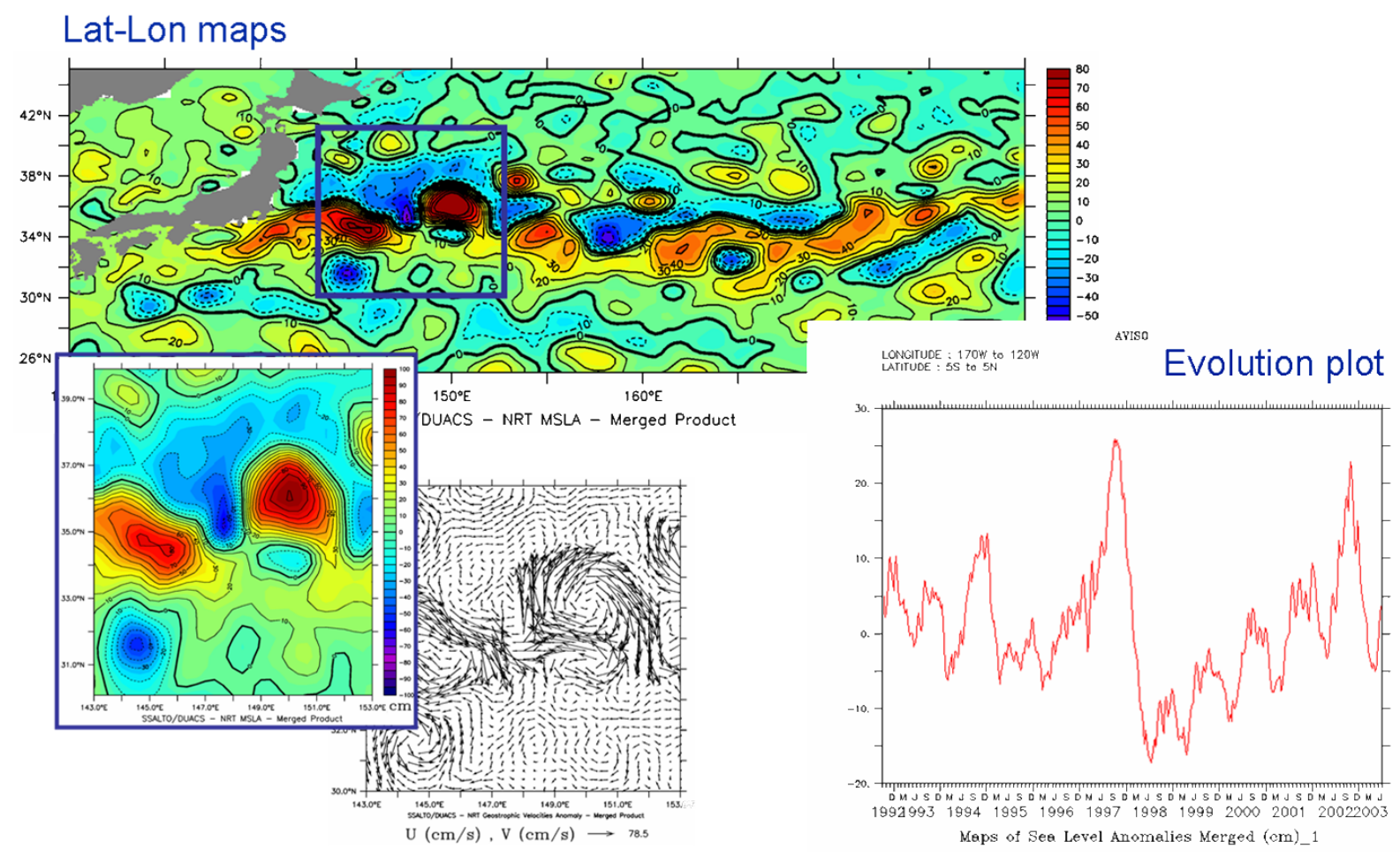

Fig. 2. Some plots made by Aviso Live Access Server. Statistical plots (average, variance), hovmoller (longitude-time) diagram are also possible, as well as comparison plots.

- request custom subsets of variables in a choice of file formats (gif, ascii, NetCDF),

- compare (difference) variables from distributed locations,

- retrieve statistical information about the data (min, max, average, variance...),

- access background reference material about the data (metadata).

Ferret is the default visualization application used by LAS, though other applications (Matlab, IDL, GrADS,...) can also be used by the Opendap (see).

\subsection{Aviso Live Access Server}

Aviso Live Access Server distributes several Ssalto/Duacs altimetry products such as delayed and near-real time sea level anomalies, absolute dynamic topography, absolute geostrophic velocities, gridded significant wave height and gridded wind speed modulus. These data are issued from a set of four altimetry satellites: GFO (Geosat Follow-On), Jason-1, Envisat, Topex/Poseidon. Merged products processed from the combination of the different satellites' data are also distributed. Long time-series are thus available, with more than twelve years of gridded sea level anomalies over the whole ocean.

\subsection{Feedback: uses \& users}

The HTTP protocol allows logs analysis of whom (by IP number), and what, is downloaded or visualized by the Opendap and Live Access Server. More than 230 different IP addresses connected in April 2005, for 8036 requests on the Live Access Server. Most users are laboratories, or scientific organization, with some few individuals connected by an Internet access provider. Opendap use is slowly increasing, with some peaks mostly due to a lone user; Opendap users are only professionals, from organizations like Noaa (USA), or Ifremer (France).

Analysing what and how people use those tools, we can discriminate between several user profiles, mainly between "data fans", mostly scientists who download a lot of data and "onlookers", who prefer to visualize one or a few plots. However, it is clear that, for now, users are mostly people who seem to know about altimetry and Aviso data, since the most accessed data are not the first ones of the list, but the best in term of quality ("merged" data, i.e. multi-missions datasets).

\section{3 "Lively data": an online tutorial for Aviso LAS}

The Live Access Server allows quite a lot of visualisation and plots, it is easier-to-use than Opendap directly, but advanced capabilities are somehow a bit hard to find and use, 
and the tool, like most, has its own quirks and some nonevident tricks. Thus we decided to propose users with a regular chronicle on Aviso web site, called "Lively data" ("Balades en LAS" in the French version) dedicated to LAS tips and tricks. For example, how to constrain minimum and maximum of the color scale, or how to do an average, how to show contours, or not, or how to compute variance, and over how large an area and how long a period it is feasible to do it, etc. Moreover, this is one more element for the web site life, always a good thing to have visitors coming back. It is also a more efficient way of promoting this tool and having people have a look at it than a one-shot announcement.

By 25 April, 2005, we published 32 pages (in both English and French, see http://www.aviso.oceanobs.com/html/ donnees/las/), making a review of the tool possibilities with oceanographic examples (see Fig. 2): modifying output options (including scale extrema, or colors), computing statistical functions, comparing data, working with time series, etc., and showing along the way all the available data.

\section{Conclusions}

Opendap/LAS are quite well adapted to scientific \& nonscientific users, but they are still a bit complicated for everybody. Features as we propose in the "Lively data" pages are useful for data users, even new to the field, but still need a bit of oceanographic background, and some knowledge of the data, and of mathematics (what are averages, variances, etc.). For a really general audience, simplification would have to be made in the list of available data, keeping only the most relevant. Some adapted preferences should be used for the plots (e.g. color scale minima and maxima, often irrelevant when automatic for altimetry data), and some options rendered unavailable. For educational use, translation would also be in order.

In that optic, too, especially for end-users and education, we plan to investigate the possible use of Opendap with a GIS (e.g. Map Server), that would require less knowledge about the data and about data plotting in general, and propose mouse-drive interactive capabilities.
Edited by: E. Cutrim, M. Ramamurthy, S. Nativi, and L. Miller

Reviewed by: anonymous referees

\section{References}

Aviso website: http://www.aviso.oceanobs.com, Aviso Live Access Server: http://las.aviso.oceanobs.com; Aviso Opendap: http: //opendap.aviso.oceanobs.com.

Baudel, S., Blanc, F., Jolibois, T., and Rosmorduc, V.: Discover, browse and access ocean data on internet, PV-2004 Ensuring the Long-Term Preservation and Adding Value to the Scientific and Technical Data, Frascati, 2004.

Live Access Server website: http://ferret.pmel.noaa.gov/Ferret/ LAS/, 10 February 2005.

Lively data website: http://www.aviso.oceanobs.com/html/ donnees/las/.

Opendap website: http://opendap.org, 10 February 2005.

Rosmorduc, V.: Aviso, at the crossroad between user service, outreach and education, Adv. Geosci., 4, 1-4, 2005. 